Original

\title{
Wireless sensor networks: A survey on monitoring water quality
}

\author{
Mompoloki Pule*, Abid Yahya, Joseph Chuma \\ Electrical, Computer and Telecommunications Engineering Department, Botswana International University of Science and Technology, Palapye, Botswana \\ Received 28 April 2016; accepted 16 July 2017 \\ Available online 3 January 2018
}

\begin{abstract}
Diseases related to poor water and sanitation conditions have over 200 million cases reported annually, causing 5-10 million deaths world-wide. Water quality monitoring has thus become essential to the supply of clean and safe water. Conventional monitoring processes involve manual collection of samples from various points in the distribution network, followed by laboratory testing and analysis. This process has proved to be ineffective since it is laborious, time consuming and lacks real-time results to promote proactive response to water contamination. Wireless sensor networks (WSN) have since been considered a promising alternative to complement conventional monitoring processes. These networks are relatively affordable and allow measurements to be taken remotely, in real-time and with minimal human intervention. This work surveys the application of WSN in environmental monitoring, with particular emphasis on water quality. Various WSN based water quality monitoring methods suggested by other authors are studied and analyzed, taking into account their coverage, energy and security concerns. The work also compares and evaluates sensor node architectures proposed the various authors in terms of monitored parameters, microcontroller/microprocessor units (MCU) and wireless communication standards adopted, localization, data security implementation, power supply architectures, autonomy and potential application scenarios.

(C) 2017 Universidad Nacional Autónoma de México, Centro de Ciencias Aplicadas y Desarrollo Tecnológico. This is an open access article under the CC BY-NC-ND license (http://creativecommons.org/licenses/by-nc-nd/4.0/).
\end{abstract}

Keywords: Real-time; Remote; Water quality monitoring; Wireless sensor networks

\section{Introduction}

Wireless sensor networks (WSNs) have gained popularity within research community because they provide a promising infrastructure for numerous control and monitoring applications. These simple low-cost networks allow monitoring processes to be conducted remotely, in real-time and with minimal human intervention. A typical WSN network consists of two main components namely node and base-station, as shown in Figure 1. A node is a device that is normally equipped with sensing, processing and communication capabilities, and is responsible for measuring the parameters associated with a particular application. A base station is responsible for capturing and providing access to all measurement data from the nodes, and can some-

\footnotetext{
* Corresponding author.

E-mail address: calvin.pule@gmail.com (M. Pule).
}

Peer Review under the responsibility of Universidad Nacional Autónoma de México. times provide gateway services to allow the data to be managed remotely (Bhende, Wagh, \& Utpat, 2014).

WSNs normally employ Wireless Personal Area Network (WPAN) or Low Power Wide Area Network (LPWAN) standards, to relay measurement data to the base station. These standards include IEEE 802.15.4, ZigBee and Bluetooth. There is no single connectivity solution considered suitable for all WSNs, and the choice of standard entirely depends on communication requirements and resource constraints of a particular application. Typical considerations for selecting a wireless connectivity solution are specified in Table 1.

\subsection{WSN communication standards}

\subsubsection{ZigBee}

ZigBee is a WPAN standard that has been layered on top of the IEEE 802.15.4 specification to include the networking and application layers. ZigBee devices are capable of creating star, cluster tree and mesh network topologies and can communicate 


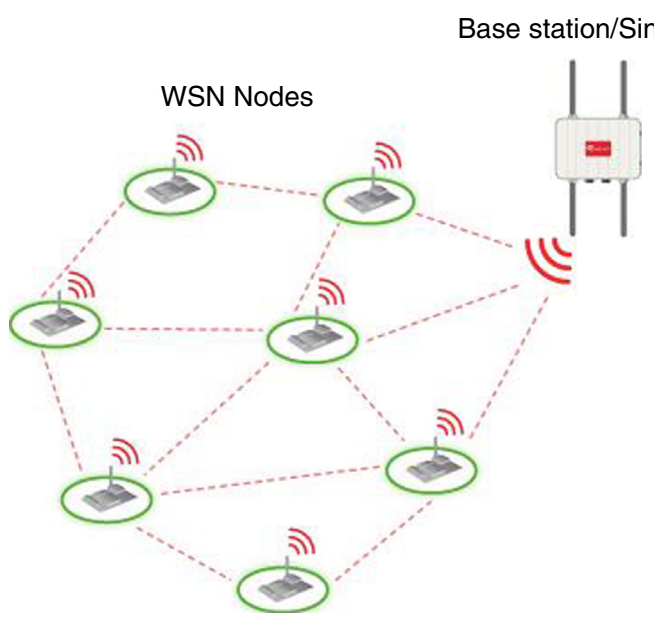

Fig. 1. A typical WSN (Libelium, 2016).

Table 1

Considerations in the selection of a WSN connectivity standard.

\begin{tabular}{|c|c|}
\hline Item & Description \\
\hline Frequency & $\begin{array}{l}\text { WSNs often employ unlicensed ISM frequency bands for } \\
\text { low cost implementation. Some of these bands are } \\
\text { vulnerable to channel blocking and signal interference } \\
\text { which is not suitable for critical application scenarios. }\end{array}$ \\
\hline Range & $\begin{array}{l}\text { In some WSN applications, nodes are deployed in remote } \\
\text { rural areas which require connectivity solutions with good } \\
\text { range capabilities. Selecting the right solution requires } \\
\text { pre-assessment of network coverage requirements. }\end{array}$ \\
\hline Data Rate & $\begin{array}{l}\text { The type and size of data to be sent across a WSN plays a } \\
\text { significant role in the choice of connectivity method. Image } \\
\text { and video signals require high data rate solutions as } \\
\text { compared to other data signals. }\end{array}$ \\
\hline Power & $\begin{array}{l}\text { Energy is a limited resource for all WSNs, but different } \\
\text { applications require different levels of autonomy and hence } \\
\text { the selected connectivity standard must satisfy the } \\
\text { associated power requirements. }\end{array}$ \\
\hline Security & $\begin{array}{l}\text { WSN connectivity standards offer different levels of } \\
\text { security. Sensors supporting mission critical applications } \\
\text { would require a careful consideration of this aspect. }\end{array}$ \\
\hline
\end{tabular}

over hundreds of meters with a maximum data rate of $250 \mathrm{kbps}$ (Gungor, Hancke, \& Member, 2009; Lee, Su, \& Shen, 2007).

\subsubsection{Bluetooth low energy (BLE)}

BLE is an ultra-low power version of the Bluetooth specification which allows data rates of up to $1 \mathrm{Mbps}$ in the $30-80 \mathrm{~m}$ range context (Gungor et al., 2009; Lee et al., 2007).

\subsubsection{Long range wide area network (LoRaWAN)}

LoRaWAN is a technology that has been designed for applications that need to send small amounts of data over long distances a few times per day. Its low power features offers the capability to achieve autonomy of up to 10 years (LoRa Alliance \& Machina Research, 2015; LoRa Alliance \& Technical Marketing Workgroup, 2015; LoRa Alliance, 2015a, 2015b).

\subsubsection{SigFox}

SigFox is the world's first cellular network dedicated to low bandwidth Machine-to-Machine and Internet of Things applications. Its patented Ultra Narrow Band (UNB) technology utilizes unlicensed frequency bands to transmit data over a very narrow spectrum. Sigfox has a range capability of up to $40 \mathrm{~km}$ in open space (SigFox, n.d.).

Table 2 provides a technical comparison of the commonly employed WSN standards.

\section{Applications of WSN}

WSNs have numerous applications ranging from military surveillance, industrial monitoring, medical telemetry and environmental monitoring. These applications have different operational requirements, which is why they tend to adopt different WSN architectures. For military surveillance, the most important requirements include high bandwidth, high security and good coverage. Industrial monitoring applications require secure, reliable, robust and real-time WSN solutions. Medical applications often put more emphasis on security and network reliability, and environmental monitoring usually requires robust, energy efficient and autonomous nodes (Bhende et al., 2014; Buratti, Conti, Dardari, \& Verdone, 2009; Girão, Postolache, \& Pereira, 2014; Zhao, 2011).

\subsection{Environmental monitoring}

The development of human society has come with major impacts on the environment, and all efforts to improve its conservation have been aggressively sought. Environmental monitoring is one such significant effort, which has allowed various physical parameters to be monitored in order to control or limit further progression of environmental degradation. Conventional monitoring techniques required manual collection of environmental data, but were later considered inefficient since they are labor intensive and lack early warning capability to issues of environmental contamination. Some years ago, digital data loggers were introduced to help improve the spatial and temporal resolution of environmental monitoring, but still lacked real-time data analytics. With the advent of micro-electromechanical-systems (MEMS), low power WSN technologies were developed, and environmental monitoring could be conducted remotely and in real-time (Oliveira \& Rodrigues, 2011). This approach has since promoted pro-active response to environmental contamination.

\subsubsection{Air quality monitoring}

Han and Cui (Zhi-gang \& Cai-hui, 2009) reviewed the application of ZigBee based WSN in monitoring air pollution. The proposed system employed GPS enabled sensor nodes to monitor air quality parameters and transmit them to a sink node linked to a computer network. GIS analysis was suggested to simulate spatial and temporal distribution of air pollution in a given area. However, the proposed schema was only developed for a small area, which implies additional networking 
Table 2

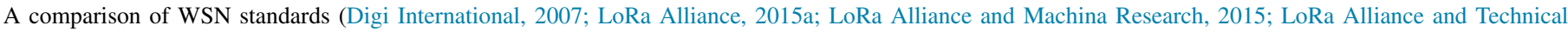
Marketing Workgroup, 2015; SigFox, n.d.).

\begin{tabular}{|c|c|c|c|c|c|}
\hline Wireless standards & Frequency band (MHz) & Data rates (kbps) & Range capability (LoS) & Network topology & Comparative power consumption \\
\hline IEEE 802.15 .4 & $2400,915,868$ & $250,40,20$ & $100 \mathrm{~m}+$ & Star & Low \\
\hline ZigBee & $2400,915,868$ & $250,40,20$ & $100 \mathrm{~m}+$ & Star, tree and mesh & Low \\
\hline Bluetooth & 2400 & 720 & $10-100 \mathrm{~m}$ & $\mathrm{P} 2 \mathrm{P}$ and star & High \\
\hline LoRaWAN & 915,868 & 50 & $10 \mathrm{~km}+$ & Star & Very low \\
\hline SigFox & 902,868 & 1 & $40 \mathrm{~km}$ & Star & Very low \\
\hline
\end{tabular}

requirements will have to be taken into account when considering a relatively large area.

\subsubsection{Forest monitoring}

Jadhav and Deshmukh (2012) designed and developed a WSN system for detecting forest fires by monitoring environmental parameters such as temperature, smoke and humidity. The system employed LPC $2138 \mathrm{MCU}$ powered nodes to process measurement data and transmit it to a base-station using ZigBee. From experimental testing, the system was able to take readings and send them for real-time sensor data logging. Nonetheless, the system was battery operated and did not integrate any energy scavenging mechanisms such as solar to extend the lifetime of the nodes.

\subsubsection{Climatological monitoring}

Keshtgari (2012) designed a WSN system to monitor climatological and environmental parameters for use in precision agriculture. A system of 24 nodes was modeled employing OPNET and its performance was investigated under two grid ZigBee topologies. From simulation results, the proposed methodology achieved high transmission success rates proving great potential for employing ZigBee technology in agricultural systems. Nevertheless, the adopted topologies were not optimized for power consumption and therefore limit the WSN lifetime.

Other applications of WSN in environmental monitoring include earthquake/seismic detection, landslide and avalanche detection, snow level monitoring and water quality monitoring.

\section{Monitoring water quality with WSN}

Water borne diseases have become a major challenge to human health. Around 250 million cases of such diseases are reported annually, causing 5-10 million deaths world-wide (Chung \& Yoo, 2015). Therefore, ensuring water quality is undoubtedly highly significant to maintaining a sustainable civilization for all. Water quality can be defined as the suitability of water for a particular application based on its chemical, biological and physical characteristics. Monitoring water quality involves detecting its characteristic parameters and comparing them with set standards and guidelines. Acceptability of the water is normally determined by compliance with the standards of its intended usage (Gorchev \& Ozolins, 1984). One of the biggest challenges in monitoring water quality is the requirement to collect a large number of samples in order to ensure accurate and reliable analysis. In this regard, manual methods introduce considerable complexity and are considered ineffective. Real-time monitoring enables early warning capability to ensure timely response to water contamination. This is why WSNs have been thoroughly investigated for this application. Additionally, these networks require relatively low startup and maintenance costs, and therefore show great potential for numerous monitoring applications.

Monitoring water quality with WSN employs nodes that can detect physico-chemical parameters such as $\mathrm{pH}$, conductivity, turbidity and chlorine.

\section{Common physico-chemical water quality parameters}

i. $\mathrm{pH}$

This is a measure of the degree of acidity or alkalinity of a solution. A pH of 7 is neutral, while that below and above neutral is considered acidic and alkaline, respectively. For distribution systems, a pH that is between 6.0 and 9.0 is normally recommended.

ii. Electrical conductivity

This is a measure of a solution's capability to pass electrical current. Conductivity is used as an indication of the concentration conductive ions that normally come from dissolved salts and other inorganic material. The more the dissolved salts present, the higher the conductivity. Drinking water should have a conductivity that ranges between 0 and $2500 \mu \mathrm{S} / \mathrm{cm}$.

iii. Oxidation reduction potential (ORP)

ORP measures how strongly electrons are transferred between component species in a solution. This indicates the ability of water to rid itself of contaminants. Healthy water normally has very high ORP readings.

iv. Turbidity

This indicates the concentration of suspended and colloidal material in water and it is measured in nephelometric turbidity units (NTU). Drinking water should have turbidity that is less than 1 NTU.

\subsection{Challenges of monitoring water quality with WSN}

Monitoring water quality is critical to human health, hence employing WSN for such a task requires a system that is robust, secure and has a reliable communication. 


\subsubsection{Energy resources}

Shao, Liu, and Zhang (2012) designed and developed a WSN system for monitoring industrial sewerage based on ARM-7 LPC2138 powered sensor nodes. Authors achieved a communication range of up to $1.5 \mathrm{~km}$ with a power output of $+25.99 \mathrm{dBm}$. Nonetheless, using the ARM-7 microprocessor is costly since it runs an operating system in addition to executing the application code. This introduces additional computational complexity which leads to high power consumption. Furthermore, the system adopts a dynamic routing backup mechanism, which also introduces processing and memory/storage overheads, leading to additional power requirements. Khetre and Hate (2013) designed a WSN system for monitoring the quality of lake waters consisting of ARM 7 slave nodes for measuring water quality parameters. A two-slave prototype was developed and employed ZigBee to transmit data to a master terminal. The system was tested on turbid water and a reading of $30.6 \mathrm{NTU}$ was recorded. All other parameters were captured on a live graph.

Vijayakumar and Ramya (2015) designed and developed a low cost, real time water quality monitoring system using Internet of Things (IoT) technologies. The node was powered with a Raspberry Pi model B+ microprocessor, with several water quality sensors connected to it. From experimental results, the proposed system was able to present water quality parameters on the Internet. However, monitoring water quality is a process of relative low complexity to require the functionality of advanced microprocessors proposed by the authors (Khetre \& Hate, 2013; Vijayakumar \& Ramya, 2015). With the Raspberry Pi solution, energy resources will be depleted rapidly since the microprocessor has to run a LINUX operating system in addition to executing the application code. Furthermore, cloud computing requires implementation of security mechanisms since it exposes sensor data to potential cyber-attacks, and in turn results in additional computational, bandwidth, memory and energy costs.

Chung and Yoo (2015) designed a WSN system to detect water pollution in rivers, streams and coastal areas. The system employed TesloB series ATmega128 microcontroller powered sensor nodes. A data averaging algorithm was designed to reduce the size of the communication traffic, and a TinyOS query-based flooding routing protocol was used to transfer data through the network. From experimental results, the authors indicated that the averaged water quality data was transmitted every $5 \mathrm{~min}$ interval and the data transmission loss ratio was below $1 \%$. Even so, the communication reliability offered by a flooding scheme introduces additional processing, information overheads which lead to huge costs in terms of energy, memory and bandwidth. This is inefficient for WSNs since they have limited resources. Table 3 compares nodes architectures of reviewed WSN water quality monitoring solutions with energy concerns.

\subsubsection{Security}

Yue and Ying (2012) presented a novel water quality monitoring system made up of several solar powered sensor nodes, which employ IEEE 802.15.4 to communicate with a base station. The authors developed a prototype with one node, based on a solar powered SunSPOT WSN transceiver, and a base station. The prototype was tested on 6 water samples by measuring their $\mathrm{pH}$ and turbidity values and was able to provide real time feedback. He and Zhang (2012) designed a two component WSN based water quality monitoring system for the environmental protection department. The system employed a ZigBee network of CC2430 MCU nodes and an internet based remote data center accessible via a GPRS DTU. Authors estimated the node's current consumption to be less than $110 \mu \mathrm{A}$ and would achieve a lifetime of up to six months or more. However, the proposed system does not provide any local data storage, which implies that water quality information will not be accessible where there is no Internet connection.

Nasser, Ali, Karim, and Belhaouari (2013) designed and implemented an energy efficient, self-configurable and reusable WSN-based water quality monitoring system. The proposed framework consists of open hardware ZigBee sensor motes (Squidbee), a data center that is connected to the Internet, and a fixed client-server component that includes an information portal and a web server. Table 4 compares nodes architectures of reviewed WSN water quality monitoring solutions with data security concerns.

A prototype was designed based on the proposed architecture, and from experimental results authors indicated that the system can monitor water quality in real-time and has a lifetime that is longer than typical WSN frameworks. Lambrou, Anastasiou, Panayiotou, and Polycarpou (2014) designed and developed a low cost in-pipe sensor network system for monitoring drinking water quality at consumer sites. The system node and base station employed PIC32 and ARM/Linux microcontroller platforms, respectively. From experimental results, authors have shown that the system is capable of detecting some high impact microbiological (Escherichia coli) and chemical (Arsenic) contaminants at fairly low concentrations. However, monitoring all parameters using a single node is risky since a nodal malfunction will lead to loss of all water quality data.

All the authors in work (He \& Zhang, 2012; Lambrou et al., 2014; Nasser et al., 2013; Vijayakumar \& Ramya, 2015; Yue \& Ying, 2012) proposed remote access to the system via the Internet without properly addressing security concerns. This approach introduces vulnerabilities that can affect authenticity, integrity and confidentiality of measurement data as a result of cyber-attacks.

\subsubsection{Coverage and connectivity}

O'Flynn et al. (2007) developed "SmartCoast", a multi sensor system for monitoring water quality based on the Tyndall motes that employed ZigBee for wireless connectivity. Each node was enclosed in an IP68 waterproof case and could monitor key water quality parameters such as temperature, $\mathrm{pH}$, conductivity, depth, turbidity, phosphate and dissolved oxygen. From results, the authors were able to validate the viability of a low power multi sensor WSN implementation. Wang, Wang, and Hao (2009) developed a remote water quality monitoring system made up of a multi-hop self-organized ZigBee network for measuring water quality parameters, a control center computer to provide storage, management and data visualization services, and an internet connection to allow remote access to water quality data from anywhere, respectively. GPRS was employed to 
Table 3

A comparison of proposed node architectures for water quality monitoring systems with energy concerns.

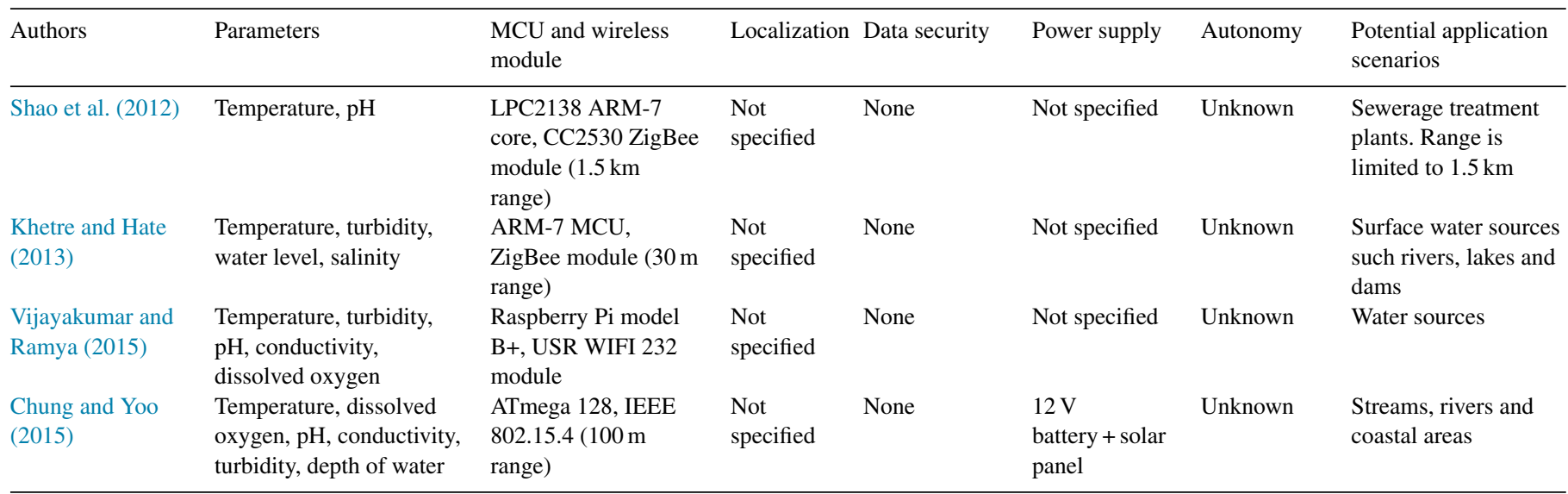

Table 4

A comparison of proposed node architectures for water quality monitoring systems with data security concerns.

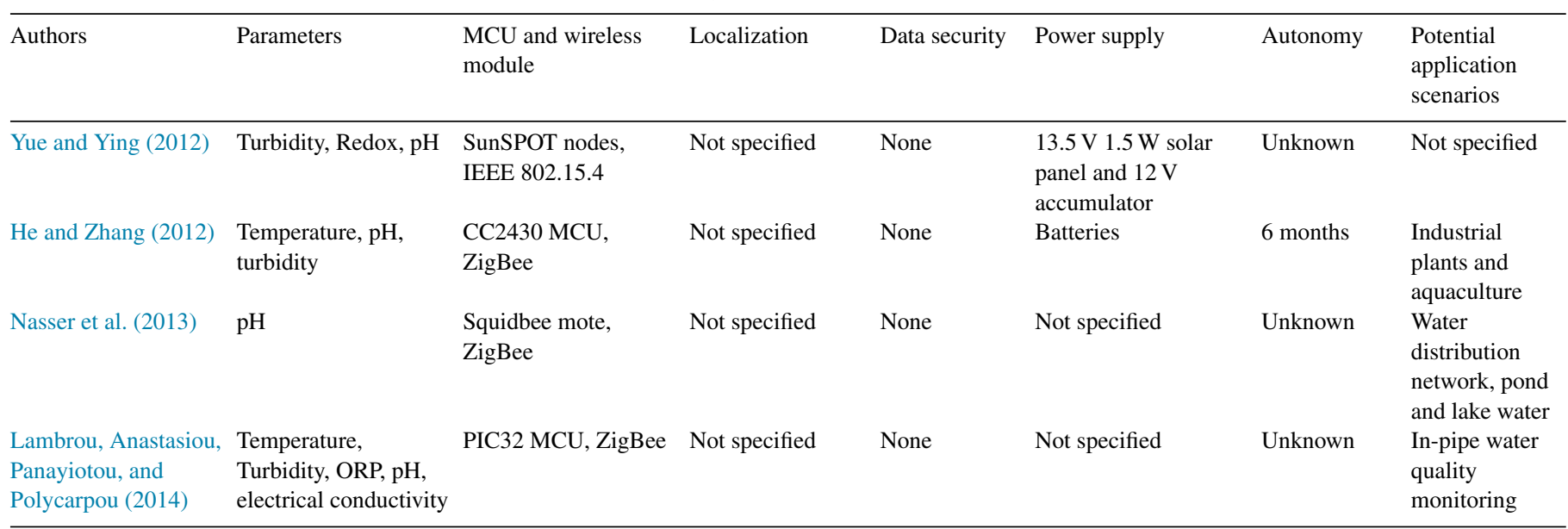

achieve long distance monitoring. The authors highlighted that experimental tests confirm a stable system operation.

Rasin and Abdullah (2009) proposed a ZigBee based WSN system for monitoring water quality. A GUI platform was developed at the base station using Borlan $\mathrm{C}++$ builder to visualize the water quality data. From experimental results, the nodes consumed $35.5 \mathrm{~mA}$ and $28.8 \mathrm{~mA}$ in active and sleep modes, respectively. Jiang, Xia, He, and Wang (2009) designed a WSN water environment monitoring system that sampled $\mathrm{pH}$ and temperature measurements every hour and transmitted the data to a base station using the ZigBee. GPRS was used for communication between the base station and an online remote monitoring center. Authors indicated that the system was able to perform measurements ranging from 0 to $80 \pm 0.5^{\circ} \mathrm{C}$ and 0 to $14 \pm 0.05$ for temperature and $\mathrm{pH}$, respectively. However, the two parameters presented are not sufficient for microbial analysis, which is normally indicated by ORP, turbidity and chlorine measurements.

Zennaro et al. (2009) proposed an integrated sensor network system for monitoring water quality in Malawi. A 90-FLT series E sensor board was used to measure the selected water quality parameters and SunSPOT sensor motes were then used to establish an IEEE 802.15.4 WSN network to send the measurements to a multi-platform ALIX 2 gateway. Huang, Chou, Jau, and Sandnes (2010) designed a system for monitoring water quality with ubiquitous computing employing a hand-held water quality meter coupled with RS232 and 3G network interface card. From experimental results, authors verified the applicability of the proposed system to monitor water quality with reduced requirements of device deployment. Nevertheless, a hand-held monitoring device affects the ability of a system to operate autonomously and unattended, and any malfunction of the device leads to loss or corruption of all water quality data.

Zhu et al. (2010) developed a system that employs CDMA with IPsec-based Virtual Private Network (VPN) to monitor and forecast water quality in modern intensive fish farming in China. Forecasting was implemented through Artificial Neural Networks (ANN). Authors deployed and tested a prototype system of two nodes for 22 months, and were able to achieve statistical reliability of $95.2 \%$. However, the use of $\mathrm{WiFi}$ for local data collection imposes high power requirements on sensor nodes and reduces their lifetime. Zhang and Wang (2011) developed a real time water quality monitoring WSN system for aquaculture. The data acquisition nodes use ZigBee to send 
the collected data to router nodes, which then employ GSM to forward it to the monitoring systems. The WSN system was deployed in crab ponds of local farmers at Yixing Jiangsu province, China.

Nasirudin, Za'bah, and Sidek (2011) described an approach to real-time fresh water quality monitoring using WSN. The proposed system employs PIC16F886 MCU based solar powered nodes, to collect and send water quality data to a coordinator/base-station via ZigBee. The base station allows remote data access with a server PC equipped with a GSM modem. From experimental results, authors were only able to test, successfully, the system functionality of a few prototypes that could measure water level and temperature for both the WSN (ZigBee) and GSM systems. Ji, Ji, and Zhang (2011) designed a GPRS-based real-time water quality monitoring system composed of GPS enabled nodes, each powered by a 32-bit Cortex-M3 microcontroller and a GPRS module. From experimental testing and debugging, authors highlighted that the system was able to achieve a stable performance and reliable operation. Nonetheless, GPRS technology is not really optimized for WSN applications and it has more power requirements than typical WSN standards such as IEEE 802.15.4 and ZigBee.

Amruta and Satish (2013) proposed an underwater WSN system for monitoring water quality. A prototype was developed with one node powered by a solar cell, employing ZigBee to send $\mathrm{pH}$, turbidity and oxygen level sensor data to a base station. From experimental results, a VB 6.0 GUI was used to analyze water quality data, and graphical results were generated hourly using MATLAB. Nevertheless, the prototype system allowed access to water quality data by direct connection to the base station and offered no means of remote management to promote proactive response to water contamination. Karthikeyan, Geethanjali, Mekala, and Deepika (2014) proposed a method for monitoring water quality using WSN. The suggested method employs SunSPOT piezoelectric/wind energy powered sensor nodes to collect water quality measurements and send to a base station by means of IEEE802.15.4. Faustine et al. (2014) designed and developed a solar powered system for monitoring water quality within Lake Victoria Basin using WSN. Sensor nodes used an Arduino core to process measurement data before sending it to the gateway via ZigBee. The gateway captured all the data and relayed it to the application software using GPRS. From field test results, authors demonstrated proper functionality and practical application of the proposed system in a real environment. Nevertheless, the system has no provision for local data analysis and will therefore be offline every time a mobile network outage is experienced.

Sridharan (2014) designed and developed a ZigBee networked WSN system for monitoring water quality. A MATLAB GUI was developed at the base station to visualize the water quality data. From simulation and testing, the system was able to capture and plot $\mathrm{pH}$, temperature and turbidity measurements on the GUI. Vacariu, Cret, Hangan, and Bacotiu (2015) developed a system for monitoring water quality parameters of some river waters in Romania based on WSN technology. Nodes used the IEEE 802.15.4 radio protocol to communicate sensor data to a gateway, where it was processed and transmitted to a remote storage server via GSM. Authors estimated the total cost of their system to be around 4000 USD, and further highlighted that it is cheaper than the regular discharge measuring station which costs around 70000 USD.

Adamo et al. (2015) proposed a solution for continuous space-time monitoring of surface water quality based on a network of smart sensors. A low cost sea water probe with the ability to measure chlorophyll-a, conductivity, turbidity and temperature was employed. The authors suggested that implementation of the solution would result in immediate improvement of the effectiveness of surface water quality monitoring. However, using a single probe to detect all water parameters is not very reliable since all data will be lost when the probe malfunctions.

The systems proposed by Adamo et al. (2015), Karthikeyan et al. (2014), Amruta and Satish (2013), O’Flynn et al. (2007), Rasin and Abdullah (2009), Sridharan (2014), Vacariu et al. (2015), Wang et al. (2009), Zennaro et al. (2009) employ either IEEE 802.15.4 or ZigBee for communication between the nodes and base-station. However, these technologies have a range that is limited to only a few tens of meters which limits spatial resolution of the system. The range is usually extended by employing additional relay nodes, which tend to introduce additional routing costs. These technologies normally operate in the $2.4 \mathrm{GHz}$ ISM license free band which is often crowded and vulnerable to interference and security attacks. Authors of (Adamo et al., 2015; Huang et al., 2010; Ji et al., 2011; Jiang et al., 2009; Nasirudin, Za'bah, \& Sidek, 2011; Vacariu et al., 2015; Zhang \& Wang, 2011; Zhu et al., 2010) employed the mobile network as part of the WSN architecture to allow remote access to the system. However, this approach will incur additional communication costs/charges as the network expands since it relies on operator based infrastructure. Furthermore, the system will inherit all the limitations of the associated mobile network thereby disturbing WSN autonomy. Table 5 compares nodes architectures of some of reviewed WSN water quality monitoring solutions with coverage and connectivity concerns.

\section{Conclusion}

Wireless sensor networks offer a promising infrastructure to municipal water quality monitoring and surveillance. Their greatest benefit comes from affordability and the ability to conduct measurements remotely and in real-time. However, these networks have resource limitations in terms of processing power, memory, communication bandwidth and energy/power. If not addressed properly, these limitations can hinder the effectiveness and efficiency of employing WSN in monitoring applications. Most of the previously adopted WSN water quality monitoring architectures had limited range capabilities, and to improve their coverage they had to employ repeater nodes and complex routing algorithms while others relied heavily on the mobile network. Even so, such approaches introduced additional networking and computational costs which put a burden on resource limited WSN nodes. Some architectures employed cloud computing for remote access to water quality data but did not address security concerns. Nonetheless, this approach threatens the reliability of the system as it leaves sensor data vulnerable to cyber-attacks. 
Table 5

A comparison of proposed node architectures for water quality monitoring systems with coverage and connectivity concerns.

\begin{tabular}{|c|c|c|c|c|c|c|c|}
\hline Authors & Parameters & $\begin{array}{l}\text { MCU and wireless } \\
\text { module }\end{array}$ & Localization & Data security & Power supply & Autonomy & $\begin{array}{l}\text { Potential application } \\
\text { scenarios }\end{array}$ \\
\hline $\begin{array}{l}\text { Rasin and Abdullah } \\
\text { (2009) }\end{array}$ & $\begin{array}{l}\text { Temperature, } \mathrm{pH}, \\
\text { turbidity }\end{array}$ & $\begin{array}{l}\text { TI } 8051 \mathrm{MCU}, \mathrm{TI} \\
\text { CC2430 ZigBee } \\
\text { module }\end{array}$ & Not specified & None & $9 \mathrm{~V}$ battery & $12 \mathrm{~h}$ & $\begin{array}{l}\text { Natural water } \\
\text { resources }\end{array}$ \\
\hline Zennaro et al. (2009) & $\begin{array}{l}\text { Temperature, } \mathrm{pH} \text {, } \\
\text { conductivity, total } \\
\text { dissolved solids } \\
\text { (TDS), dissolved } \\
\text { oxygen, turbidity }\end{array}$ & $\begin{array}{l}\text { SunSPOT nodes, } \\
\text { IEEE } 802.15 .4 \text { radio }\end{array}$ & Not specified & None & $\begin{array}{l}3.7 \mathrm{~V} 720 \mathrm{mAh} \\
\text { battery }\end{array}$ & 14 days & Surface water sources \\
\hline Zhu et al. (2010) & $\begin{array}{l}\text { Temperature, } \mathrm{pH}, \\
\text { dissolved oxygen, } \\
\text { conductivity, salinity }\end{array}$ & $\begin{array}{l}\text { PICNIC } 2.0 \text { core } \\
\text { processing chip, } \\
\text { CDMA module }\end{array}$ & Not specified & IPsec VPN & Not specified & $\begin{array}{l}\text { Tested over a } 2 \text { year } \\
\text { period. Autonomy } \\
\text { unknown }\end{array}$ & Fish culture \\
\hline Nasirudin et al. (2011) & $\begin{array}{l}\text { Temperature, } \mathrm{pH}, \\
\text { turbidity, dissolved } \\
\text { oxygen }\end{array}$ & $\begin{array}{l}\text { PIC16F886 MCU, } \\
\text { IEEE 802.15.4 }\end{array}$ & Not specified & None & $\begin{array}{l}12 \mathrm{~V} \text { battery pack, } \\
\text { solar panel }\end{array}$ & Unknown & $\begin{array}{l}\text { Fresh water sources } \\
\text { such as rivers, } \\
\text { streams, lakes }\end{array}$ \\
\hline $\begin{array}{l}\text { Amruta and Satish } \\
\text { (2013) }\end{array}$ & $\begin{array}{l}\mathrm{pH} \text {, oxygen level, } \\
\text { turbidity }\end{array}$ & $\begin{array}{l}\text { LPC } 2148 \text { ARM7 } \\
\text { MCU, ZigBee module }\end{array}$ & Not specified & None & $\begin{array}{l}13.5 \mathrm{~V} 1.5 \mathrm{~W} \text { solar } \\
\text { panel, } 12 \mathrm{~V} \text { battery }\end{array}$ & Unknown & Surface water sources \\
\hline $\begin{array}{l}\text { Karthikeyan et al. } \\
\text { (2014) }\end{array}$ & $\begin{array}{l}\mathrm{pH} \text {, turbidity, } \\
\text { dissolved oxygen }\end{array}$ & $\begin{array}{l}\text { SunSPOT nodes, } \\
\text { ZigBee module }\end{array}$ & Not specified & None & $\begin{array}{l}\text { Wind power piezo } \\
\text { electric power } \\
\text { module, } 9 \mathrm{~V} \text { battery }\end{array}$ & Unknown & Surface water sources \\
\hline Faustine et al. (2014) & $\begin{array}{l}\text { Temperature, } \mathrm{DO}, \mathrm{pH} \text {, } \\
\text { conductivity }\end{array}$ & $\begin{array}{l}\text { Arduino Mega 2560, } \\
\text { ZigBee }\end{array}$ & GPS (EM-506) & None & $\begin{array}{l}3.7 \mathrm{~V} 6 \mathrm{AH} \text { battery and } \\
10 \mathrm{~W} \text { solar panel }\end{array}$ & Unknown & $\begin{array}{l}\text { Water sources, } \\
\text { treatment plant and } \\
\text { distribution network }\end{array}$ \\
\hline
\end{tabular}


In future, this work plans to develop a prototype water quality monitoring system employing LPWAN standards for their low power consumption and improved range capabilities. The system will in addition take into account data security requirements for water quality surveillance. Power optimization techniques will also be investigated to improve the autonomy and life-time of sensor nodes.

\section{Conflict of interest}

The authors have no conflicts of interest to declare.

\section{References}

Adamo, F., Attivissimo, F., Guarnieri, C., Carducci, C., Maria, A., \& Lanzolla, L. (2015). . pp. 2514-2522. A smart sensor network for sea water quality monitoring (Vol. 15(5))

Amruta, M. K., \& Satish, M. T. (2013). Solar powered water quality monitoring system using wireless sensor network. In International multi-conference on automation, computing, communication, control and compressed sensing (iMac4s) (pp. 281-285). IEEE. http://dx.doi.org/10.1109/iMac4s.2013.6526423

Bhende, M., Wagh, S. J., \& Utpat, A. (2014). A quick survey on wireless sensor networks. In Proceedings - 2014 4th international conference on communication systems and network technologies, CSNT 2014 (pp. 160-167). http://dx.doi.org/10.1109/CSNT.2014.40

Buratti, C., Conti, A., Dardari, D., \& Verdone, R. (2009). An overview on wireless sensor networks technology and evolution. Sensors (Basel, Switzerland), 9(9), 6869-6896. http://dx.doi.org/10.3390/s90906869

Chung, W.-Y., \& Yoo, J.-H. (2015). Remote water quality monitoring in wide area. Sensors and Actuators B: Chemical, 217, 1-7. http://dx.doi.org/10.1016/j.snb.2015.01.072

Digi International. (2007). Demystifying 802.15.4 and ZigBee.. Retrieved from: http://www.mouser.com/pdfdocs/digi-wp_zigbee.pdf

Faustine, A., Mvuma, A. N., Mongi, H. J., Gabriel, M. C., Tenge, A. J., \& Kucel, S. B. (2014, December). Wireless sensor networks for water quality monitoring and control within lake victoria basin: Prototype development. pp. 281-290. http://dx.doi.org/10.4236/wsn.2014.612027

Gorchev, H. G., \& Ozolins, G. (1984). WHO guidelines for drinkingwater quality. WHO Chron, 38(3), 104-108. http://dx.doi.org/ 10.1016/S1462-0758(00)00006-6

Gungor, V. C., Hancke, G. P., \& Member, S. (2009). Industrial wireless sensor networks: Challenges, design principles, and technical approaches. IEEE Transactions on Industrial Electronics, 56(10), 4258-4265.

He, D., \& Zhang, L.-X. (2012). The water quality monitoring system based on WSN. In 2012 2nd international conference on consumer electronics, communications and networks (CECNet) (pp. 3661-3664). http://dx.doi.org/10.1109/CECNet.2012.6201666

Huang, Y.-P., Chou, C.-T., Jau, J.-S., \& Sandnes, F.-E. (2010). Water quality monitoring with ubiquitous computing. In 20107 th international conference on ubiquitous intelligence \& computing and 7 th international conference on autonomic \& trusted computing (UIC/ATC), vol. 1 (pp. 70-75). http://dx.doi.org/10.1109/UIC-ATC2010.17

Jadhav, P. S., \& Deshmukh, V. U. (2012). Forest fire monitoring system based on ZIG-BEE wireless sensor network. International Journal of Emerging Technology and Advanced Engineering, 2(12), 187-191.

Ji, B., Ji, S., \& Zhang, C. (2011). GPRS-based data real-time transmission system of water-quality monitoring. In 2011 international conference on electronics, communications and control (ICECC) (pp. 2072-2075).

Jiang, P., Xia, H., He, Z., \& Wang, Z. (2009). Design of a water environment monitoring system based on wireless sensor networks. Sensors, 9(8), 6411-6434. http://dx.doi.org/10.3390/s90806411

Karthikeyan, V., Geethanjali, S., Mekala, M., \& Deepika, T. (2014). Water eminence scrutinizing scheme based on zigbee and wireless antenna expertise - A study. International Journal of Scientific Research in Computer Science Applications and Management Studies, 3(1), 1-5.

Keshtgari, M. (2012). A wireless sensor network solution for precision agriculture based on zigbee technology. Wireless Sensor Network, 4(1), 25-30. http://dx.doi.org/10.4236/wsn.2012.41004

Khetre, A. C., \& Hate, S. G. (2013). Automatic monitoring \& reporting of water quality by using WSN technology and different routing methods. International Journal of Advanced Research in Computer Engineering \& Technology, 2(12), 3255-3260.

Lambrou, T. P., Anastasiou, C. C., Panayiotou, C. G., \& Polycarpou, M. M. (2014). A low-cost sensor network for real-time monitoring and contamination detection in drinking water distribution systems. IEEE Sensors Journal, 14(8), 2765-2772. http://dx.doi.org/10.1109/JSEN.2014.2316414

Lee, J., Su, Y., \& Shen, C. (2007). A comparative study of wireless protocols: Bluetooth, UWB, ZigBee, and Wi-Fi. In 33rd annual conference of the IEEE industrial electronics society (IECON) (pp. 46-51).

Libelium. (2016). Libelium Website. Retrieved from: http://www.libelium.com

LoRa Alliance. (2015a). LoRaWAN TM Specification.. Retrieved from: https://www.lora-alliance.org/portals/0/specs/LoRaWAN_Specification 1R0.pdf

LoRa Alliance. (2015b). Wide Area Networks for IoT.. Retrieved from: https://www.lora-alliance.org/lorawan-white-papers

LoRa Alliance \& Machina Research. (2015). LPWA Technologies.. Retrieved from: https://docs.wixstatic.com/ugd/eccc1a_a45b82212b3e4a 3098cc714200e8e5c6.pdf

LoRa Alliance \& Technical Marketing Workgroup. (2015). A technical overview of LoRa ${ }^{\circledR}$ and LoRaWANTM.. Retrieved from: https://docs.wixstatic.com/ugd/eccc1a_ed71ea1cd969417493c74e4a13 c55685.pdf

Nasirudin, M. A., Za'bah, U. N., \& Sidek, O. (2011). Fresh water realtime monitoring system based on Wireless Sensor Network and GSM. In 2011 IEEE conference on open systems (ICOS) (pp. 354-357). http://dx.doi.org/10.1109/ICOS.2011.6079290

Nasser, N., Ali, A., Karim, L., \& Belhaouari, S. (2013). An efficient Wireless Sensor Network-based water quality monitoring system. In 2013 ACS international conference on computer systems and applications (AICCSA) (pp. 1-4). http://dx.doi.org/10.1109/AICCSA.2013.6616432

O’Flynn, B., Martínez, R., Cleary, J., Slater, C., Regan, F., \& Murphy, H. (2007). SmartCoast: A wireless sensor network for water quality monitoring. In 32nd IEEE conference on local computer networks (pp. 815-816). http://dx.doi.org/10.1109/LCN.2007.34

Oliveira, L. M., \& Rodrigues, J. J. (2011). Wireless sensor networks: A survey on environmental monitoring. Journal of Communications, 6(2), 143-151. http://dx.doi.org/10.4304/jcm.6.2.143-151

Rasin, Z., \& Abdullah, M. R. (2009). Water quality monitoring system using zigbee based wireless sensor network. International Journal of Engineering \& Technology, 9(10), 14-18.

Shao, X., Liu, X., \& Zhang, H. (2012). Monitoring system of sewerage treatment based on wireless network. In 2012 international conference on optoelectronics and microelectronics (ICOM) (pp. 490-492).

SigFox. (n.d.). SigFox Website. Retrieved from: http://www.sigfox.com.

Silva Girão, P., Postolache, O., \& Pereira, J. D. (2014). Wireless sensor network-based solution for environmental monitoring: Water quality assessment case study. IET Science, Measurement \& Technology, 8(6), 610-616. http://dx.doi.org/10.1049/iet-smt.2013.0136

Sridharan, S. (2014). Water quality monitoring system using wireless sensor network. International Journal of Advanced Research in Electronics and Communication Engineering (IJARECE), 3(4), 399-402.

Vacariu, L., Cret, O., Hangan, A., \& Bacotiu, C. (2015). Water parameters monitoring on a cyberwater platform. In 2015 20th international conference on control systems and computer science (pp. 797-802). http://dx.doi.org/10.1109/CSCS.2015.24

Vijayakumar, N., \& Ramya, R. (2015). The real time monitoring of water quality in IoT environment. In IEEE sponsored 2nd international conference on innovations in information, embedded and communication systems (ICIIECS) 2015 (pp. 1-4).

Wang, Z., Wang, Q., \& Hao, X. (2009). The design of the remote water quality monitoring system based on WSN. In 5th international conference on wire- 
less communications, networking and mobile computing, 2009. WiCom '09 (pp. 1-4). http://dx.doi.org/10.1109/WICOM.2009.5303974

Yue, R., \& Ying, T. (2012). A novel water quality monitoring system based on solar power supply \& wireless sensor network. Procedia Environmental Sciences, 12(Icese 2011), 265-272. http://dx.doi.org/10.1016/j.proenv.2012.01.276

Zennaro, M., Floros, A., Dogan, G., Sun, T., Cao, Z., Huang, C., ..., \& Bagula, A. (2009). On the design of a water quality wireless sensor network (WQWSN): An application to water quality monitoring in Malawi. In 2009 international conference on parallel processing workshops (pp. 330-336). http://dx.doi.org/10.1109/ICPPW.2009.57

Zhang, M. F., \& Wang, L. Z. (2011). A WSN-based monitor system for water quality combined with expert knowledge. In 2011 international conference on electronics, communications and control, ICECC 2011 - Proceedings (pp. 105-108). http://dx.doi.org/10.1109/ICECC.2011.6067719

Zhao, G. (2011). Wireless sensor networks for industrial process monitoring and control: A survey. Network Protocols and Algorithms, 3(1), 46-63. http://dx.doi.org/10.5296/npa.v3i1.580

Zhi-gang, H., \& Cai-hui, C. (2009). The application of zigbee based wireless sensor network and GIS in the air pollution monitoring. In 2009 international conference on environmental science and information application technology (pp. 546-549). http://dx.doi.org/10.1109/ESIAT.2009.192

Zhu, X., Li, D., He, D., Wang, J., Ma, D., \& Li, F. (2010). A remote wireless system for water quality online monitoring in intensive fish culture. Computers and Electronics in Agriculture, 71(Suppl. 1), 3-9. http://dx.doi.org/10.1016/j.compag.2009.10.004 